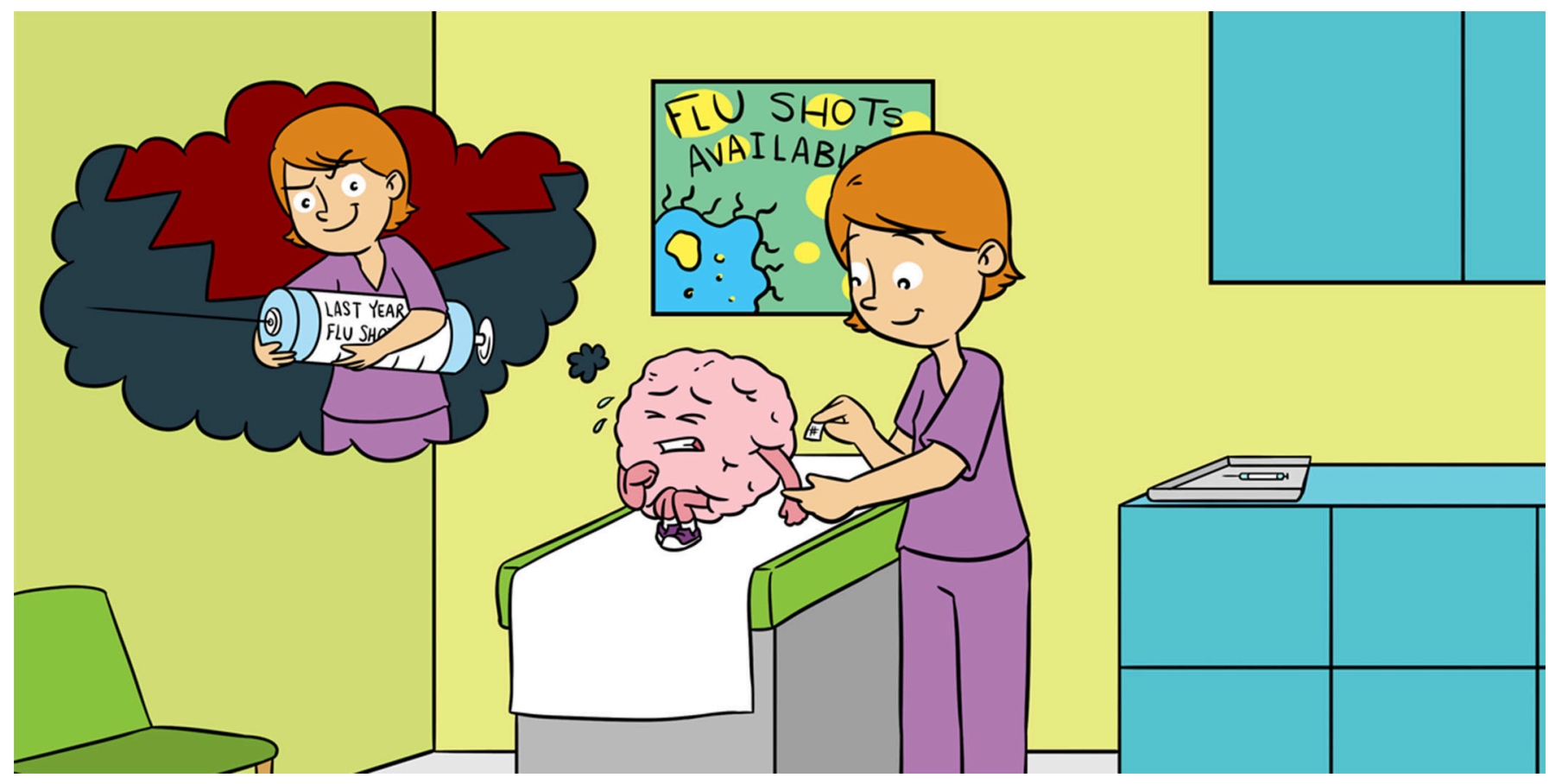

\title{
THIS WILL ONLY HURT FOR A MINUTE: HOW OUR BRAINS PLAN FOR PAIN
}

\section{Giovanna Del Sordo ${ }^{1}$, Emma Moyer ${ }^{2}$, Dre Goode ${ }^{3}$ and Michael C. Hout ${ }^{3,4^{*}}$}

${ }^{1}$ Acquisition of Understanding, Knowledge, and Skills Laboratory, Department of Psychology, New Mexico State University, Las Cruces, NM, United States

${ }^{2}$ Hearing Enhancement and Augmented Reality Laboratory, Department of Psychology, New Mexico State University, Las Cruces, NM, United States

${ }^{3}$ Vision Sciences and Memory Laboratory, Department of Psychology, New Mexico State University, Las Cruces, NM, United States

${ }^{4}$ Addison Care Virtual Reality and Augmented Reality Laboratory, New Mexico State University, Las Cruces, NM, United States

YOUNG REVIEWERS:

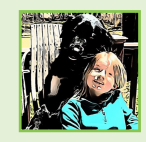

KENLEY

AGE: 8

PARKER

AGE: 10
Pain is something we all experience, but how does it work? Pain is best understood as an unpleasant sensation that causes physical discomfort. The sensation of pain works as an alarm system, telling us when we should avoid certain activities or when we should be mindful of body parts that may have experienced damage. People experience pain in a variety of ways, sometimes describing it as "sharp," "shooting," "aching," and feeling it in other ways that are often hard to define. Many things besides the actual cause of pain impact our expectations and experiences of pain, such as our personality traits, previous experiences, and prior discussions about pain. What we feel depends on both physical sensations and our expectations: anticipation can sometimes make our pain sensations worse. However, with some positive thinking, you can actually 


\section{make yourself experience less pain by controlling how you think an experience will make you feel.}

\section{PAIN EXPECTATION}

A belief about what might happen in the future, specifically concerning pain experiences.

\section{SUBJECTIVE}

\section{MEASURES}

Measurements that rely on what people say they experienced. For example, the question "How much does it hurt" on a scale from 0 "not at all" to 10 "worst pain possible?" is a subjective measure because it only asks the person to say what they felt.

\section{OBJECTIVE} MEASURES

Measurements that use instruments to assess something more directly, and do not rely on a person's report of their experience. For example, using fMRI to detect if brain areas involved with pain are activated is an objective measure because a person cannot control his/her brain activation.

\section{NEUROIMAGING}

Methods that either produce images of the brain's structure or look at the brain's activity.

\section{WHAT IS PAIN?}

Pain quickly directs our attention to an area of the body where we might be injured or be experiencing something negative. This is why pain can be a great alarm system: because we quickly become aware of the physical sensation and try to stop whatever is causing it. Pain is a physical sensation that we experience, but that sensation is also influenced by our thoughts and feelings. These thoughts and feelings are directly related to our previous pain experiences and our memories and emotions about these encounters. If we have sad and upsetting memories about pain, they can make our physical sensations of pain worse. Equally, less sad memories can reduce the physical sensation of pain. This moderating effect of our memories and feelings is what we call our pain expectation. Being aware of our pain expectations can help us be in charge of our pain, allowing us to keep control over our thoughts and feelings instead of letting our pain control the way we react. Though pain is an important alarm system for most of us, some people are born with an insensitivity to it, which causes them to not feel pain at all. This is dangerous because these individuals are not aware of being burned, breaking a bone, or other things that pain tries to warn us to take care of. So, we do not want to experience a complete absence of pain in our lives; instead, we want to have a healthy understanding of the role of pain and how to properly manage it.

\section{HOW DO WE STUDY PAIN?}

Studying pain is a pain! In the past, researchers have had to study pain using subjective measures, but in recent years, more objective measures have been developed from tools that produce images of the brain and its activity, called neuroimaging. By subjective measures, we mean any information or data that we cannot measure directly, but rather rely on someone to report back to us. "How much did that hurt?" is a subjective question that we ask in pain research, but we cannot directly measure how much something hurts. Instead, we ask people to tell us how painful something was. Subjective measures can be tricky for researchers, because the questions researchers ask can be interpreted differently from one person to the next. Objective measures, on the other hand, allow us to use tools or devices to record information directly, and to compare it across different people. Advances in technology that have allowed us to take pictures of the brain and measurements of its activation have provided us with new, objective measures of pain and pain expectation. These methods can be very expensive, so researchers and clinicians still often rely on subjective measures. Many methods can be used to create pain for 
research purposes, such as heat, cold water, or even mild electric stimulation of the hand. These methods, though painful, are safe when they are used for short periods of time and in a way that does not actually damage the skin or any part of the body.

\section{WHAT IS PAIN EXPECTATION?}

Expectations change the way that we perceive pain [1]. An expectation is a belief about what might happen in the future. Our expectations concerning a situation come from what we have experienced before, or from what other people have told us about what happened to them in a similar situation. We can have both positive and negative expectations. For example, you may have positive expectations - that make you feel happy or excited-about your upcoming birthday because, in previous years, you received wonderful gifts. Alternatively, you may have negative expectations-that make you feel scared, angry, or sad-about events, such as going to the dentist. This is how pain expectation works: if your last trip to the dentist was painful (maybe you had to get a filling) you may anticipate pain on the next trip. But if that prior experience was not too bad (maybe you just had a cleaning), then you may anticipate a pleasant experience on the next visit. These expectations about pain can greatly influence the ways that we think and behave in a certain situation and can make the situation better or worse for us. Let us look at a specific example to explain how pain expectations work.

\section{MANIPULATION OF PAIN EXPECTATION}

Researchers have shown that pain expectation is something that can be controlled. In one study (see Figure 1), scientists tricked participants into expecting more pain than they actually felt [2]. They did this by first holding a training session, in which participants had the pain device attached to their legs. Researchers would wait for a short, medium, or long time, and then the pain device would heat up to a temperature that was either a bit hot $\left(114^{\circ} \mathrm{F}\right)$, more hot $\left(118^{\circ} \mathrm{F}\right)$, or very hot $\left(122^{\circ} \mathrm{F}\right)$. Then the participants had to rate how painful the temperature was. As expected, the participants rated the hotter temperatures as more painful than the temperatures that were less hot. By doing this training session, the researchers got the participants to associate longer wait times with hotter temperatures (and greater pain!). Participants came back for an experimental session, in which the amount of time spent waiting and the temperature of the pain device were not connected at all, but participants still thought they were. So, when participants waited for a long time but only felt the temperature that was a bit hot, they still thought it was the very hot temperature. The expectation made them feel more pain than the stimulus was actually causing! 
Figure 1

During training (left), participants waited for a short, medium, or long time and then felt a temperature that was either a bit hot, more hot, or very hot.

Participants thus linked longer wait times with more painful

experiences. During the experiment (right), the amount of time waiting and the temperature were not connected at all, but participants still thought they were. If participants then waited for a long time but only felt the temperature that was a bit hot, they still thought it was the very hot temperature and they overestimated the pain. If they waited for a short time, and felt the very hot temperature, they thought it was only a bit hot and they underestimated the pain.

fMRI

A tool which make it possible to look at the brain's activity while performing a task, and to detect which brain's area is more active during a certain task.

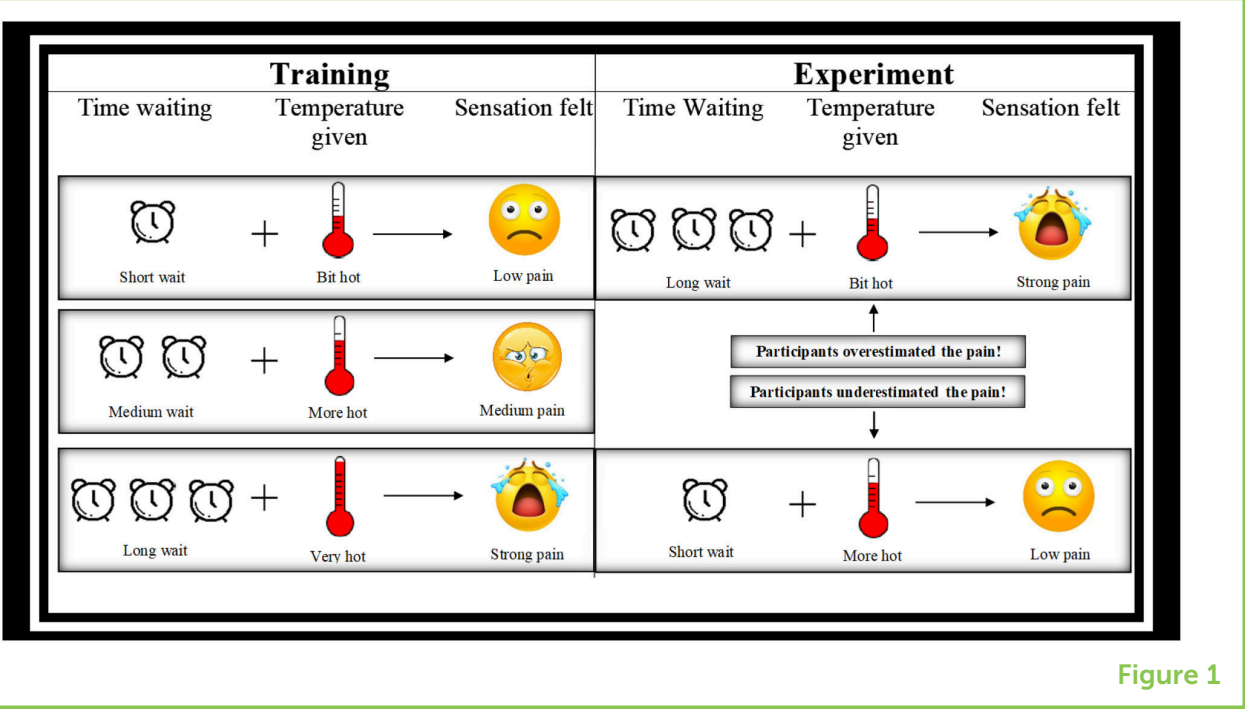

The researchers also used a neuroimaging technique called functional magnetic resonance imaging (fMRI) in their study. Scientists use fMRI to study brain activity. Your brain and muscles work in similar ways: they both require energy. Oxygen is carried by your blood to different areas of your brain. This oxygen supplies the brain with the energy that it needs to function. When using an fMRI scanner, scientists identify brain activity by recording changes in the oxygen levels of the blood that is in the brain. The more that a certain brain area is being used, the more oxygen it will need. The fMRI scanner shows us which areas of the brain are using more oxygen, and therefore, which areas of the brain are being used more heavily.

The researchers used fMRI to look at brain areas known to be involved in pain expectation and pain experience. The brain areas related to pain showed the most activation when participants expected and experienced the most pain from the very hot temperature, and the smallest amount of activation when participants expected and experienced the least pain from the temperature that was a bit hot. However, the pain areas of the brain also showed lots of activation when the participants were expecting the very hot temperature, even if they only felt the cooler temperatures! In the same way that the participants rated their pain as worse when they were expecting worse pain, their brains also reacted as if they were experiencing the worst pain just because they were expecting that kind of pain.

\section{POSITIVE AND NEGATIVE EXPECTATIONS}

It should be becoming clear that it is possible to develop positive or negative expectations regarding pain. Think about the last time you got a flu shot (see Figure 2). There were likely many aspects of the doctor's office that caused you to brace for a certain amount of pain: the cleaning of your skin, the presentation of the needle, etc. These 
Figure 2

How does pain expectation work? The way we remember and prepare for pain can change how we experience it. When you feel pain, if you decide that it is not very scary and not that bad, the next time you are in a similar situation, you may have positive expectations and feel less pain than you did before. But on the contrary, if you experience pain and are afraid and exaggerate all your negative feelings, you might worsen your memory for pain and feel even more pain in the future.

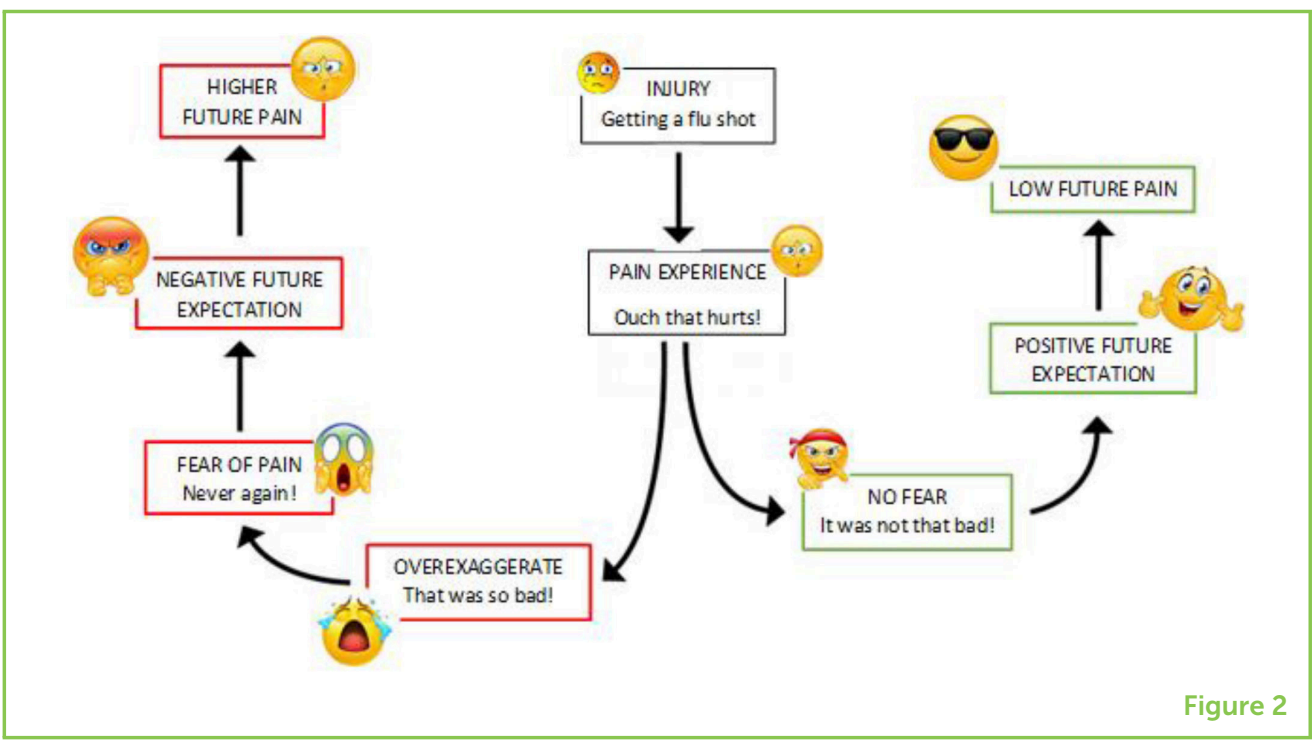

painful events later prompt us with reminders about the level of pain that others have shared with us, as well as our own previous memories of painful experiences. The way we remember and prepare for pain can greatly impact and modify the pain we feel. Depending on the way we believe the experience is going to feel, we can increase or decrease the sensations we will actually experience. Once you get the shot and realize it is not that bad, you can retain a memory that it was okay after all. By remembering that the next time you visit the doctor, you can carry your positive expectations with you and feel less pain than you would have if you had negative expectations.

On the contrary, negative expectations can lead to worse experiences in the future [3]. If, for instance, you are fearful of shots, you may exaggerate those experiences in your memory and set yourself up for more pain on future visits. The key here is the way you think about the pain you are going to encounter. Next time you know that you are going to experience something painful, try to go into the situation with a positive attitude; that way you can try to lessen the pain sensations you actually feel.

\section{CONCLUSION}

Being in pain is not enjoyable, so we do not look forward to any situation in which we anticipate the experience of pain. But while negative expectations can make our experience of pain worse, positive expectations can make things better, or at least not as bad! Remember, to some degree we can control how we feel, so being positive can have beneficial effects on our lives in all sorts of areas, like taking tests, acting in a play, or even playing sports. Pain is just one of many things we should try to stay positive about. Make sure to remember that the next time you visit the dentist! 


\section{REFERENCES}

1. Hoskin, R., Berzuini, C., Acosta-Kane, D., El-Deredy, W., Guo, H., and Talmi, D. 2019. Sensitivity to pain expectations: a Bayesian model of individual differences. Cognition 182:127-39. doi: 10.1016/j.cognition.2018.08.022

2. Koyama, T., McHaffie, J. G., Lorienti, P. J., and Coghill, R. C. 2005. The subjective experience of pain: where expectations become reality. Proc. Natl. Acad. Sci. U.S.A. 102:12950-5. doi: 10.1073/pnas.0408576102

3. Vervoort, T., Goubert, L., Eccleston, C., Bijttebier, P., and Crombez, G. 2006. Catastrophic thinking about pain is independently associated with pain severity, disability, and somatic complaints in school children and children with chronic pain. J. Pediatr. Psychol. 31:674-83. doi: 10.1093/jpepsy/jsj059

SUBMITTED: 02 March 2020; ACCEPTED: 27 August 2020; PUBLISHED ONLINE: 14 October 2020.

EDITED BY: Fanli Jia, Seton Hall University, United States

CITATION: Sordo GD, Moyer E, Goode D and Hout MC (2020) This Will Only Hurt for a Minute: How Our Brains Plan for Pain. Front. Young Minds 8:539649. doi: 10. 3389/frym.2020.539649

CONFLICT OF INTEREST: The authors declare that the research was conducted in the absence of any commercial or financial relationships that could be construed as a potential conflict of interest.

COPYRIGHT () 2020 Sordo, Moyer, Goode and Hout. This is an open-access article distributed under the terms of the Creative Commons Attribution License (CC BY). The use, distribution or reproduction in other forums is permitted, provided the original author(s) and the copyright owner(s) are credited and that the original publication in this journal is cited, in accordance with accepted academic practice. No use, distribution or reproduction is permitted which does not comply with these terms.

\section{YOUNG REVIEWERS}

\section{KENLEY, AGE: 8}

I am an 8 years old girl who likes running, driving a razor, and gymnastics. My favorite food is stuffed shells. In school I like PE and math.

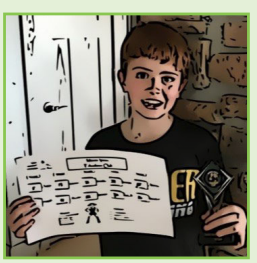

PARKER, AGE: 10

I am a 10 years old boy who really likes to do sports. My favorite is wrestling. Writing is my favorite subject in school. My hobbies include building things, sports, and driving my razor around. 

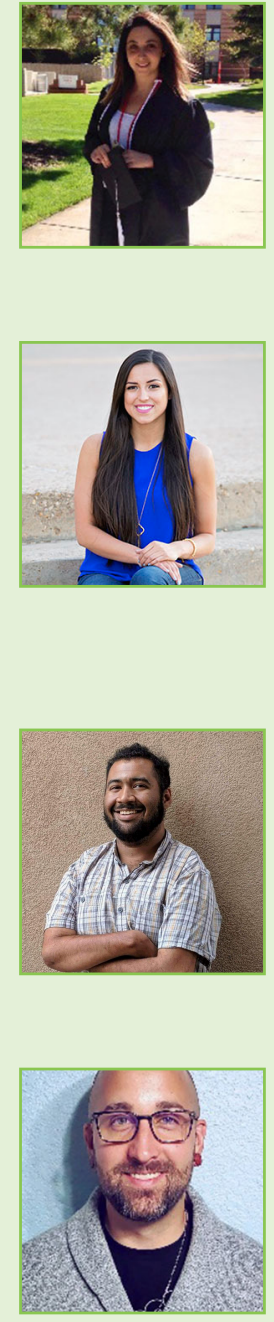

\section{AUTHORS}

\section{GIOVANNA DEL SORDO}

I am a graduate student at New Mexico State University. I am interested in studying pain and how it affects memory processes in humans. In my free time, I like to read fantasy books, to go on hikes, and to watch thriller movies.

\section{EMMA MOYER}

After receiving a Bachelor's degree in Psychology from Eastern New Mexico University, I chose to continue my psychology studies at the graduate level. I enjoy studying brain function from a neuropsychological perspective and am especially interested in researching brain function after injury. My favorite non-research activities include spending time with my friends, reading, and pursuing various athletic goals.

\section{DRE GOODE}

I am a graduate student at New Mexico State University in Las Cruces, New Mexico. I have been studying memory since the beginning of my time in college and look forward to studying how vision works with our memory to help us find things. In my free time I enjoy writing, listening to podcasts, and competitive gaming! I hope to use my interest in gaming as a platform for future research.

\section{MICHAEL C. HOUT}

Michael C. Hout is an Associate Professor in the Department of Psychology at New Mexico State University, an Associate Editor at the journal Attention, Perception, and Psychophysics, and a Program Director for the Perception, Action, and Cognition program at the National Science Foundation (in the US). His research focuses primarily on visual cognition (including visual search, attention, eye movements, and memory) and the development of alternative methods of collecting similarity data for use in multi-dimensional scaling. He has won several awards for research and teaching, including the Rising Star award from the Association for Psychological Science, and he engages in outside consulting for organizations, such as Major League Baseball. In his limited free time, he enjoys walking his dogs, hiking, playing hockey, and riding his road and mountain bikes. *mhout@nmsu.edu 